\title{
A Preliminary List of Levels and g-Values for the First Spectrum of Thorium (Th I)
}

\author{
Romuald Zalubas
}

\author{
(July 22, 1959)
}

\begin{abstract}
The present state of the analysis of the first spectrum of thorium (Th I) is discussed briefly. Even and odd levels are listed in tables 1 and 2 . The low even levels form terms arising from the configurations $6 d^{2} 7 s^{2}$ and $6 d^{3} 7 s$. The Th I standard wavelengths that fit into the known level arrays are presented in table 3.
\end{abstract}

\section{Introduction}

Work on thorium spectra was started at the National Bureau of Standards by C. C. Kiess as early as 1926. His data are included in the present work, but have never been published separately.

In 1955 it was decided to obtain a new description and analysis of the Th I spectrum at this Bureau, because electrodeless lamps were available [1]. ${ }^{1}$ The description of the thorium spectra will be published soon, and will include an account of the experimental procedures as well as the history of the thorium spectra. Therefore, only the work pertaining to the analysis of Th $\mathrm{I}$ will be mentioned here.

In $1946 \mathrm{Ph}$. Schuurmans [2] published a list of the Th I levels which he had discovered. Five of his even levels $(0.00,2869.18,5563.15,6362.39$, and $7502.25)$, and 23 of his odd levels are confirmed by the present analysis. He indicated, also, that $6 d^{2} 7 s^{2}{ }^{3} \mathbf{F}_{2}$ is the ground state of Th I. His work was based on Zeeman data for 48 Th I lines by Lier [3], and the list of Th I wavelengths by Fred [4].

\section{Procedure}

The new description of thorium spectra contains 16,000 lines between 2000 and $11500 \mathrm{~A}$. About 1,000 Zeeman patterns have been observed and measured, of which 400 are sufficiently well resolved for the determination of $g$-values. The chief difficulty in securing Th $\mathrm{I}$ Zeeman data is that the lines are heavily masked by strong Th II patterns. Therefore, two sets of plates had to be measured, one of strong and the other of weak exposures, in order to resolve a greater number of Thi patterns.

In the course of the analysis, Zeeman data from the Massachusetts Institute of Technology became available [5] for about two dozen additional lines. ThI and ThII lines have been separated by using the following criteria: (a) relative intensities in tube and spark exposures, (b) relative intensities of $n$ and $p$ components of unresolved Zeeman patterns as

\footnotetext{
1 Figures in brackets indicate the literature references at the end of this paper.
}

compared with a no-field exposure, (c) resolved Zeeman patterns, and (d) classified lines of ThII [6]. In this way 12,000 lines have been attributed to Th $\mathrm{I}$.

The vacuum wave numbers were calculated on an electronic computer by using Edlén's formula. The analysis has been substantially speeded up by the use of the computer. Bozman and Coleman [7] have written the codes with which the levels were searched by the computer from known intervals.

\section{Results}

Table 1 contains the even energy levels starting with $6 d^{2} 7 s^{2}{ }^{3} \mathrm{~F}_{2}$ as the ground state zero. The columns of this table read as follows: (1) electron configuration of the term; (2) designation of the term; (3) the inner quantum number or $J$-value of the level; (4) value of the atomic energy level in $\mathrm{cm}^{-1}$; (5) $g$-value as observed; (6) theoretical Landé $g$-value in LS-coupling. The electron configurations and term assignments of even levels appear to be fairly definite, and agree well with preliminary theoretical calculations of Trees [8].

Table 2 contains the known odd levels and has the following columns: (1) inner quantum number $J$, (2) value of energy level, and (3) observed $g$-value. Doubtful levels or those that do not have confirmation from Zeeman data are not included in this table.

The $g$-values of even levels are the means from 5 to 40 determinations, those for odd levels have from 1 to 6 determinations. The $g$-values given to three decimal places are derived from 10 or more observations.

By comparison with the ionization potentials of neighboring elements, one can expect the ionization potential of Th $\mathrm{I}$ to be between 4 and $5.5 \mathrm{ev}$. In order to establish levels of configurations $6 p^{2} 7 s n s$ and $6 d^{3} n s$ with a higher $n$-value, infrared lines beyond the photographic region must be observed.

Only a little more than half of the stronger lines are accounted for at present. The remaining lines, and about 100 well resolved Zeeman patterns indicate that there should exist another system of 
terms which is probably connected with the known levels by a small number of intercombinations. This idea is supported by the existence of two sets of terms in Th II [6]. The work on the Th I analysis will be continued in this laboratory.

\section{Secondary Standards}

Simultaneously with this work, interferometric measurements of thorium secondary standards have been made by Meggers and Stanley [9]. They have published interferometric wavelengths for 222 thorium lines of which 46 are classified as Th II and 107 are now classified as Th I. Because these lines test the accuracy of the values of energy levels, they are given in table 3 for $\mathrm{Th}$.

This investigation could not have been carried on without the help of my associates in the Spectroscopy Section. C. C. Kiess initiated the program, contributed his wavelength measurements, and has supervised the work throughout. C. H. Corliss and W. R. Bozman furnished the electrodeless tubes. The use of the digital computer for the analysis has been made possible by the help of W. R. Bozman and C. D. Coleman. R. E. Trees has assisted greatly with theoretical interpretation. It is a pleasure to express my appreciation of the very generous assistance they have all provided.

Table 1. Even energy levels of Th $\mathrm{I}$

\begin{tabular}{|c|c|c|c|c|c|c|c|c|c|c|c|}
\hline Config. & Design & $J$ & Level & Obs. $g$ & LS. $g$ & Config. & Design & $J$ & Level & Obs. $g$ & LS. $g$ \\
\hline \multirow[t]{4}{*}{$6 d^{2} \gamma_{s^{2}}$} & $a^{3} F$ & $\begin{array}{l}2 \\
3\end{array}$ & $\begin{array}{r}0.00 \\
2869.26\end{array}$ & $\begin{array}{l}0.741 \\
\text { 1. } 074\end{array}$ & $\begin{array}{l}0.667 \\
\text { 1. } 683\end{array}$ & $6 d^{3}\left({ }^{4} \mathbf{F}\right) 7 s$ & $a^{5} \mathrm{~F}$ & $\begin{array}{l}4 \\
5\end{array}$ & $\begin{array}{l}8800.25 \\
9804.81\end{array}$ & $\begin{array}{l}\text { 1. } 310 \\
\text { 1. } 366\end{array}$ & $\begin{array}{l}\text { 1. } 350 \\
\text { 1. } 400\end{array}$ \\
\hline & $a^{3} \mathrm{P}$ & $\begin{array}{l}0 \\
1 \\
2\end{array}$ & $\begin{array}{l}4961.66 \\
\\
2558.06 \\
3865.47 \\
3687.99\end{array}$ & $\begin{array}{l}\text { 1. } 212 \\
\text { 0. } 00 \\
\text { 1. } 477 \\
\text { 1. } 256\end{array}$ & $\begin{array}{r}\text { 1. } 250 \\
0 / 0 \\
\text { 1. } 500 \\
1.500\end{array}$ & $6 d^{3}\left({ }^{4} \mathrm{P}\right) 7 \mathrm{~s}$ & $a^{5} \mathrm{P}$ & $\begin{array}{l}1 \\
2 \\
3\end{array}$ & $\begin{array}{l}\text { 11601. } 03 \\
11802.94 \\
12847.97\end{array}$ & $\begin{array}{l}\text { 2. } 41 \\
\text { 1. } 721 \\
\text { 1. } 39\end{array}$ & $\begin{array}{l}\text { 2. } 500 \\
\text { 1. } 833 \\
\text { 1. } 667\end{array}$ \\
\hline & $a{ }^{1} \mathrm{D}$ & 2 & 7280.13 & 1. 189 & 1. 000 & $6 d^{3}\left({ }^{2} \mathrm{G}\right) 7 s$ & $a^{3} \mathrm{G}$ & $\begin{array}{l}3 \\
4 \\
5\end{array}$ & $\begin{array}{l}13088.57 \\
13297.42 \\
14204.30\end{array}$ & $\begin{array}{l}\text { 1. } 04 \\
0.98 \\
\text { 1. } 13\end{array}$ & $\begin{array}{l}0.750 \\
\text { 1. } 050 \\
\text { 1. } 200\end{array}$ \\
\hline & $a{ }^{1} \mathrm{G}$ & 4 & 8111.00 & 1. 08 & 1. 000 & $6 d^{3}\left({ }^{2} \mathrm{D}\right) 7 \mathrm{~s}$ & $a^{3} \mathrm{D}$ & 1 & 13962.50 & 0.76 & 0.500 \\
\hline $6 d^{\circ}\left({ }^{*} F\right) / s$ & $a{ }^{\circ} \mathrm{F}$ & $\begin{array}{l}1 \\
2 \\
3\end{array}$ & $\begin{array}{l}5563.14 \\
6362.40 \\
7502.29\end{array}$ & $\begin{array}{l}0.062 \\
\text { 1. } 014 \\
\text { 1. } 253\end{array}$ & $\begin{array}{l}\text { 1. } 000 \\
\text { 1. } 250\end{array}$ & $6 d^{3}\left({ }^{2} \mathrm{H}\right) 7 s$ & $a^{3} \mathrm{H}$ & 4 & 15493. 23 & 0.92 & 0.800 \\
\hline
\end{tabular}

TABLe 2. Odd energy levels of Th I

\begin{tabular}{|c|c|c|c|c|c|c|c|c|}
\hline$J$ & Level & Obs. $g$ & $J$ & Level & Obs. $g$ & $J$ & Level & Obs. $g$ \\
\hline 2 & 14032.10 & 1. 15 & 2 & 20922. 13 & 1. 16 & 2 & 24307. 75 & 1. 51 \\
\hline 3 & 15166. 90 & 1. 06 & 4 & 21120.45 & (1. 03$)$ & 2 & 24381. 34 & 1. 25 \\
\hline 2 & 16217.48 & 1. 10 & 3 & 21165.10 & 1. 31 & 3 & 24421. 08 & \\
\hline 3 & 16671.35 & (1. 18) & 2 & 21252.62 & 0.67 & 3 & 24561.65 & 1. 20 \\
\hline 2 & 17224.30 & 1. 07 & 4 & 21539.59 & 1. 19 & 5 & 24701.06 & 1. 15 \\
\hline 1 & 17354.64 & 0.51 & 1 & 21668. 96 & 1. 56 & 3 & 24769. 72 & 1. 15 \\
\hline 3 & 17411. 22 & 1. 12 & 2 & 21738. 04 & $(0.53)$ & 1 & 24838. 92 & 0.76 \\
\hline 2 & 17847.10 & 1. 17 & 3 & 22141.61 & 1. 10 & 3 & 24981. 10 & 1. 07 \\
\hline 4 & 18053. 64 & & 2 & 22248. 95 & 1. 13 & 3 & 25321. 95 & 1. 35 \\
\hline 3 & 18069.10 & 1. 16 & 3 & 22339.00 & 1. 01 & 4 & 25355.60 & 0.98 \\
\hline 0 & 18382.82 & 0.00 & 1 & 22396. 82 & 1. 54 & 3 & 25442.69 & 1. 10 \\
\hline 1 & 18614. 33 & 1. 41 & 2 & 22508. 06 & 1. 38 & 1 & 25526. 26 & 1. 08 \\
\hline 4 & 18809. 92 & & 3 & 22669. 90 & 1. 22 & 2 & 25703. 40 & 1. 03 \\
\hline 3 & 18930. 29 & 0.99 & 3 & 22855. 30 & 1. 09 & 1 & 25809. 30 & 1. 59 \\
\hline 2 & 19039. 15 & 1. 11 & 1 & 22877.51 & 0.64 & 4 & 25877. 52 & $(1.07)$ \\
\hline 3 & 19503.15 & 1. 10 & 1 & 23049. 46 & 1. 42 & 3 & 26036. 36 & $(0.96)$ \\
\hline 2 & 19516. 98 & 1. 37 & 2 & 23093. 98 & 1. 32 & 4 & 26048. 54 & 1. 13 \\
\hline 1 & 19817.17 & 1. 57 & 1 & 23481. 37 & 0.85 & 3 & 26096. 98 & \\
\hline 4 & 19948. 43 & (1. 29) & 3 & 23521. 06 & 1. 08 & 2 & 26113. 27 & 0.99 \\
\hline 3 & 20214. 93 & 1. 17 & 2 & 23603. 52 & 1. 39 & 1 & 26287.05 & 0.72 \\
\hline 1 & 20423.50 & 1. 42 & 4 & 23655. 16 & (1. 20) & 2 & 26363. 11 & 1. 02 \\
\hline 2 & 20522. 72 & 0.84 & 1 & 23741. 07 & $(0.71)$ & 4 & 26384. 94 & (1.07) \\
\hline 4 & 20566. 69 & & 2 & 23752.67 & 1. 11 & 3 & 26508. 03 & 1. 08 \\
\hline 0 & 20724. 37 & 0.00 & 2 & 24182.41 & $(1.27)$ & 4 & 26790.43 & 1. 14 \\
\hline 1 & 20737. 28 & 1. 42 & 4 & 24202.57 & 1. 39 & 3 & 26878.16 & 0.88 \\
\hline
\end{tabular}


TABLE 2. Odd energy levels of Th I-Continued

\begin{tabular}{|c|c|c|c|c|c|c|c|c|}
\hline$J$ & Level & Obs. $g$ & $J$ & Level & Obs. $g$ & $J$ & Level & Obs. $g$ \\
\hline 3 & 26995. 78 & 1. 18 & 3 & 29686. 37 & 1. 27 & 1 & 33161.80 & (1. 32) \\
\hline 2 & 27061.40 & $(0.94)$ & 3 & 29744.52 & 1. 06 & 4 & 33270.59 & (1. 16) \\
\hline 1 & 27087. 99 & (1. 14) & & & & 2 & 33297. 13 & (1. 23) \\
\hline 3 & 27260.17 & 1. 12 & 2 & 29853. 14 & 0. 92 & 3 & 33591. 20 & 1. 00 \\
\hline \multirow[t]{2}{*}{4} & 27266.03 & 1. 12 & 3 & 30017.10 & 1. 15 & & & \\
\hline & & & 3 & 30255.45 & 1. 12 & 3 & 33800.68 & 1. 16 \\
\hline 3 & 27317.39 & (1.07) & 1 & 30281.04 & (1. 48) & 5 & 33844.96 & 1. 10 \\
\hline 3 & $\begin{array}{l}27670.95 \\
27674.33\end{array}$ & 1. 26 & 4 & 30517.42 & 1. 46 & $\begin{array}{l}4 \\
4\end{array}$ & $\begin{array}{l}33956.93 \\
34001\end{array}$ & 10 \\
\hline $\begin{array}{l}2 \\
2\end{array}$ & $\begin{array}{l}27674.33 \\
27784.37\end{array}$ & $\begin{array}{l}1.09 \\
0.85\end{array}$ & 2 & 30553. 29 & 1.05 & $\begin{array}{l}4 \\
2\end{array}$ & $\begin{array}{l}34001.33 \\
34371.82\end{array}$ & $\begin{array}{c}1.04 \\
(1.32)\end{array}$ \\
\hline \multirow[t]{2}{*}{5} & 27852.75 & 1. 26 & 1 & 30723.82 & 1. 07 & & 34581.82 & \\
\hline & & & 3 & 30761.72 & 1. 21 & 1 & 34590.97 & (1. 48) \\
\hline 4 & 27948. 61 & 1. 28 & 2 & 30813. 00 & 0.93 & 3 & 34704.42 & (1.13) \\
\hline 1 & 28024. 69 & 1. 03 & 1 & 30928. 73 & 0. 99 & 5 & 35081.03 & (1.08) \\
\hline 2 & 28347. 55 & (1. 55) & & & & 4 & 35131.22 & 1. 26 \\
\hline 1 & 28372. 69 & 1. 79 & $\begin{array}{l}3 \\
3\end{array}$ & $\begin{array}{l}30990.52 \\
31283.12\end{array}$ & $\begin{array}{l}\text { 1. } 07 \\
1.13\end{array}$ & 5 & 35273.95 & 1. 18 \\
\hline 2 & 28513. 32 & (1. 10) & $\begin{array}{l}0 \\
3\end{array}$ & $\begin{array}{l}31283.12 \\
31523.96\end{array}$ & $\begin{array}{l}\text { 1. } 13 \\
1.11\end{array}$ & & & \\
\hline 3 & 28589. 29 & 1. 17 & 2 & 31599. 36 & 1. 18 & $\begin{array}{l}4 \\
2\end{array}$ & 35351.44 & (1.27) \\
\hline 1 & 28649.15 & 1. 11 & 1 & 31712.73 & 1. 16 & 2 & 35533.34 & 0.83 \\
\hline 3 & 28676.29 & 1. 02 & & & & $\begin{array}{l}4 \\
2\end{array}$ & $\begin{array}{l}36062.87 \\
36189.01\end{array}$ & $\begin{array}{l}1.11 \\
(1.98)\end{array}$ \\
\hline 3 & 28884.97 & 1. 17 & $\begin{array}{l}3 \\
?\end{array}$ & 31780.87 & 1. 17 & 5 & 36275.19 & $\begin{array}{l}\text { (1. } 98) \\
1.00\end{array}$ \\
\hline \multirow[t]{2}{*}{2} & 28917. 96 & 0.95 & $\begin{array}{l}2 \\
4\end{array}$ & $\begin{array}{l}31870.08 \\
31953.46\end{array}$ & $\begin{array}{l}0.93 \\
1.06\end{array}$ & & & 1.00 \\
\hline & & & 1 & 32080.39 & 0.80 & 1 & 36361.49 & 1. 11 \\
\hline 4 & 28932. 65 & 1. 09 & 3 & 32197. 12 & 1. 13 & 4 & 36382. 66 & $(1.07)$ \\
\hline 5 & 29050.77 & 1. 14 & & & & 5 & 36837. 96 & 1. 18 \\
\hline 1 & 29157. 10 & 0.89 & 3 & 32285. 23 & (1. 09) & 3 & 36871. 99 & \\
\hline 3 & 29157. 88 & 1. 17 & 4 & 32439. 05 & (1. 20) & 5 & 37008. 75 & 1. 12 \\
\hline \multirow[t]{2}{*}{1} & 29197. 33 & 1. 16 & 2 & 32575.41 & 0.78 & & & \\
\hline & & & 1 & 32665.59 & $(0.83)$ & 2 & 37149.18 & 1. 05 \\
\hline 2 & 29252. 82 & 1. 00 & 4 & 32862.51 & (1. 16) & 4 & 37605. 80 & 1. 09 \\
\hline 2 & $\begin{array}{l}29419.25 \\
20640.28\end{array}$ & $\begin{array}{l}\text { 1. } 78 \\
0.98\end{array}$ & & & & 3 & 38216.95 & \\
\hline 1 & 29640. 28 & 0.98 & 3 & 33043.35 & 1. 15 & 3 & 39611.56 & $(1.25)$ \\
\hline
\end{tabular}

TABLE 3. Classified standards of Th I

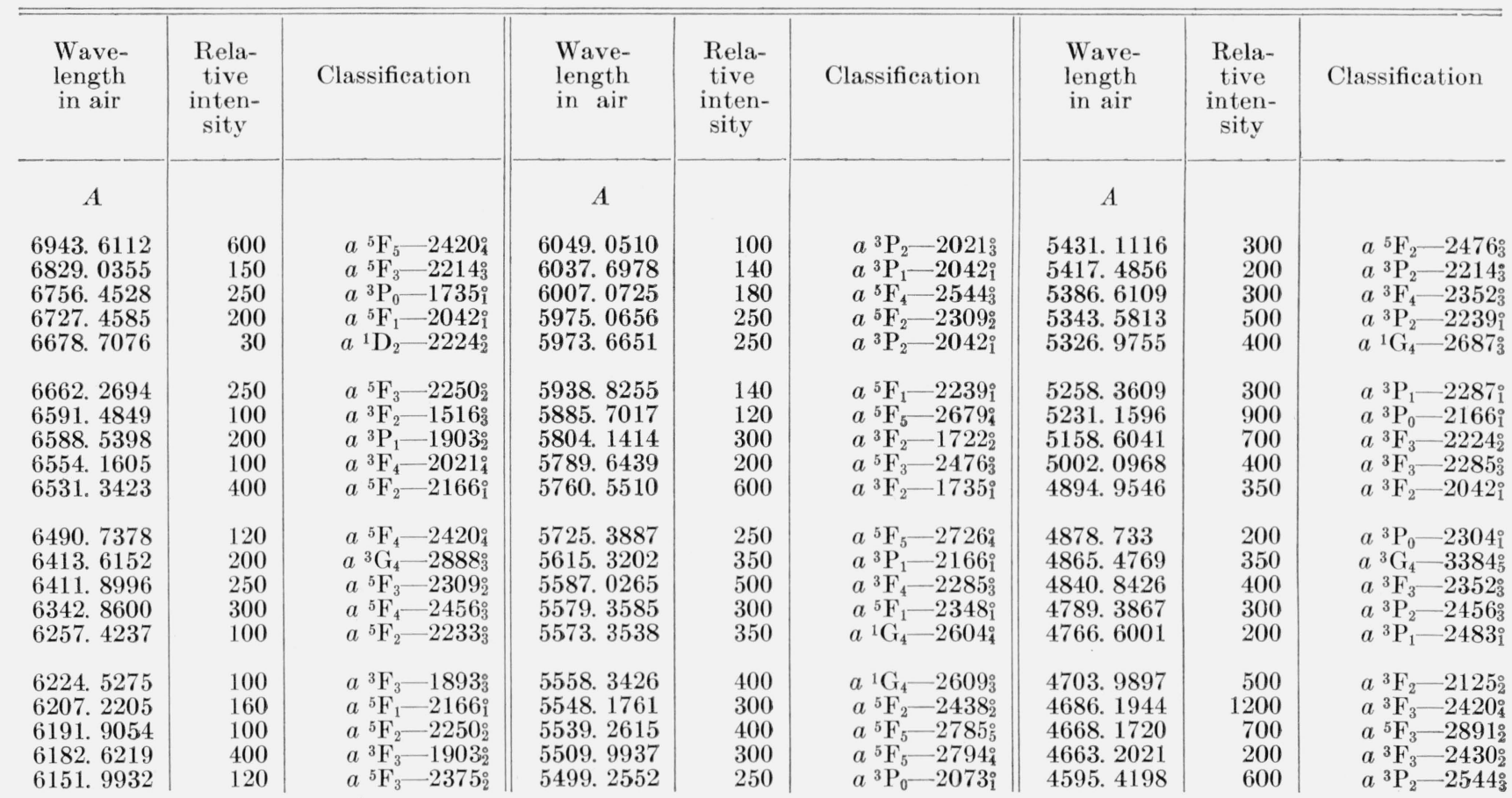


TABLE 3. Classified standards of Th I-Continued

\begin{tabular}{|c|c|c|c|c|c|c|c|c|}
\hline $\begin{array}{l}\text { Wave- } \\
\text { length } \\
\text { in air }\end{array}$ & $\begin{array}{l}\text { Rela- } \\
\text { tive } \\
\text { inten- } \\
\text { sity }\end{array}$ & Classification & $\begin{array}{l}\text { Wave- } \\
\text { length } \\
\text { in air }\end{array}$ & $\begin{array}{l}\text { Rela- } \\
\text { tive } \\
\text { inten- } \\
\text { sity }\end{array}$ & Classification & $\begin{array}{l}\text { Wave- } \\
\text { length } \\
\text { in air }\end{array}$ & $\begin{array}{l}\text { Rela- } \\
\text { tive } \\
\text { inten- } \\
\text { sity }\end{array}$ & Classification \\
\hline$A$ & & & $A$ & & & $A$ & & \\
\hline 4555.813 & $\begin{array}{r}500 \\
1200\end{array}$ & $a^{3} \mathrm{P}_{1}-2580_{1}^{\circ}$ & 4043. 3945 & $\begin{array}{r}800 \\
800\end{array}$ & $a^{3} \mathrm{~F}_{4}-2968_{3}^{\circ}$ & 3642.2487 & 2200 & $a^{3} \mathrm{~F}_{2}-2744_{2}^{\circ}$ \\
\hline $\begin{array}{l}4493.3335 \\
4482.1694\end{array}$ & $\begin{array}{r}1200 \\
300\end{array}$ & $\begin{array}{l}a^{3} \mathrm{~F}_{2}-2224_{2}^{\circ} \\
a^{3} \mathrm{~F}_{4}-2726^{\circ}\end{array}$ & $\begin{array}{l}4036.0475 \\
4012.4950\end{array}$ & $\begin{array}{l}1800 \\
2000\end{array}$ & $\begin{array}{l}a^{3} \mathrm{~F}_{2}-2476_{3}^{\circ} \\
a^{3} \mathrm{~F}_{3}-2778^{\circ}\end{array}$ & 3622.7951 & 800 & $a^{3} \mathrm{P}_{2}-3128_{3}^{\circ}$ \\
\hline 4458.0018 & 600 & $a^{3} \mathrm{P}_{2}-2611_{2}^{\circ}$ & 1010. 1000 & & & 3612.4271 & 1400 & $a^{3} \mathrm{~F}_{2}-2767_{2}^{\circ}$ \\
\hline 4408. 8828 & 600 & $a^{3} \mathrm{P}_{2}-2636_{2}^{\circ}$ & 3923. 7993 & 400 & $a^{3} \mathrm{~F}_{3}-2834_{2}^{\circ}$ & 3598. 1196 & 2000 & $a^{3} \mathrm{~F}_{2}-2778_{2}^{\circ}$ \\
\hline & & & 3869. 6635 & 600 & $a^{5} \mathrm{~F}_{2}-3219_{3}^{\circ}$ & 3584.1753 & 800 & $a^{3} \mathrm{~F}_{3}-3076_{3}^{\circ}$ \\
\hline 4378. 1768 & 500 & $a^{3} \mathrm{~F}_{3}-2570_{2}^{\circ}$ & 3839. 6941 & 2500 & $a^{3} \mathrm{~F}_{2}-2603_{3}^{\circ}$ & 3576.5573 & 1000 & $a^{1} \mathrm{G}_{4}-3606_{4}^{\circ}$ \\
\hline 4374. 124.4 & 600 & $a^{3} \mathrm{~F}_{2}-2285_{3}^{\circ}$ & 3828. 3845 & 3200 & $a^{3} \mathrm{~F}_{2}-2611_{2}^{\circ}$ & 3567,2635 & 1200 & $a^{3} \mathrm{~F}_{2}-2802_{1}^{\circ}$ \\
\hline 4315. 2544 & 400 & $a^{3} \mathrm{~F}_{3}-2603_{3}^{\circ}$ & 3803.0750 & 4000 & $a^{3} \mathrm{~F}_{2}-2628_{1}$ & 3544.0176 & 1500 & $a^{5} \mathrm{~F}_{4}-3700_{5}^{\circ}$ \\
\hline $\begin{array}{l}4257.4959 \\
4235.4635\end{array}$ & 700 & $a^{3} \mathrm{~F}_{2}-2348_{1}^{\circ}$ & $3771 \quad 3703$ & 1500 & & 3518.4033 & 1000 & $a^{3} \mathrm{~F}_{3}-3128_{3}^{\circ}$ \\
\hline 4235.4635 & 600 & $a^{3} \mathrm{~F}_{2}-2360_{2}^{\circ}$ & 3762.9345 & $\begin{array}{l}1500 \\
1200\end{array}$ & $a^{3} \mathrm{P}_{2}-3025_{3}^{\circ}$ & 3451. 7019 & 900 & $a^{3} \mathrm{P}_{2}-3265_{1}^{\circ}$ \\
\hline 4208. 8907 & 3000 & $a^{3} \mathrm{~F}_{2}-2375_{2}^{\circ}$ & 3727.9022 & 800 & $a^{3} \mathrm{~F}_{3}-2968_{3}^{\circ}$ & 3442.5785 & 800 & $a^{3} \mathrm{~F}_{4}-3400_{4}^{\circ}$ \\
\hline 4193. 0165 & 900 & $a^{1} \mathrm{G}_{4}-3195^{\circ}$ & 3719.4345 & 3000 & $a^{3} \mathrm{~F}_{2}-2687_{3}^{\circ}$ & 3405.5575 & 1400 & $a^{3} \mathrm{P}_{2}-3304_{3}^{\circ}$ \\
\hline 4158. 5351 & $\begin{array}{l}800 \\
800\end{array}$ & $a^{5} \mathrm{~F}_{5}-3384_{5}^{\circ}$ & 3700.9780 & 300 & $a^{5} \mathrm{~F}_{1}-3257_{2}^{\circ}$ & 3396.7273 & 1400 & $a^{3} \mathrm{P}_{1}-3329_{2}^{\circ}$ \\
\hline 4115.7587 & 800 & $a^{5} \mathrm{~F}_{1}-2985_{2}^{\circ}$ & & & & 3380.8595 & 900 & $a^{3} \mathrm{~F}_{3}-3243_{4}^{\circ}$ \\
\hline 4100.3412 & 1100 & $a^{3} \mathrm{~F}_{2}-2438_{2}^{\circ}$ & 3692.5661 & 1200 & $a^{3} \mathrm{P}_{2}-3076_{3}^{\circ}$ & 3330.4765 & 1800 & $a^{3} \mathrm{~F}_{2}-3001_{3}^{\circ}$ \\
\hline 4067.4507 & 400 & $a^{3} \mathrm{~F}_{3}-2744_{2}^{\circ}$ & $\begin{array}{l}3682.4861 \\
3669.9687\end{array}$ & $\begin{array}{r}1000 \\
750\end{array}$ & $\begin{array}{l}a^{3} \mathrm{~F}_{3}-3001_{3}^{\circ} \\
a^{1} \mathrm{G}_{4}-3535_{4}^{\circ}\end{array}$ & 3309.3650 & 800 & $a^{3} \mathrm{~F}_{2}-3020_{2}^{\circ}$ \\
\hline 4059.2525 & 1000 & $a^{5} \mathrm{~F}_{2}-3099_{3}^{\circ}$ & 3656.6936 & 1000 & $a^{3} \mathrm{~F}_{3}-3020_{2}^{c}$ & 3304.2381 & 3000 & $a^{3} \mathrm{~F}_{2}-3025_{3}^{\circ}$ \\
\hline
\end{tabular}

\section{References}

[1] C. C. Kiess, R. Zalubas, Spectroscopy Symposium at Argonne National Laboratory (1956); J. Opt. Soc. Am. 49, 512 (1959).

[2] Ph. Schuurmans, Dissertation, Amsterdam (1946).

[3] J. N. Lier, Dissertation Univ., Amsterdam (1939).

[4] M. S. Fred, Astrophys. J. 87, 176 (1938).

[5] See G. W. Charles, A compilation of data on some spectra of thorium, ORNL-2319 (1958).

[6] J. R. MeNally, Jr., G. R. Harrison, and H. B. Park, J. Opt. Soc. Am. 32, 334 (1942) ; J. R. MeNally, Jr., J. Opt. Soc. Am. 35, 390 (1945).
[7] W. R. Bozman and C. D. Coleman, J. Opt. Soc. Am. 49, 511 (1959).

[8] R. E. Trees, Private communication, unpublished material (1959)

[9] W. F. Meggers and R. W. Stanley, J. Research NBS 61, 95 (1958) RP2891.

Washington, D. C. 